\title{
Biographies of Contributors
}

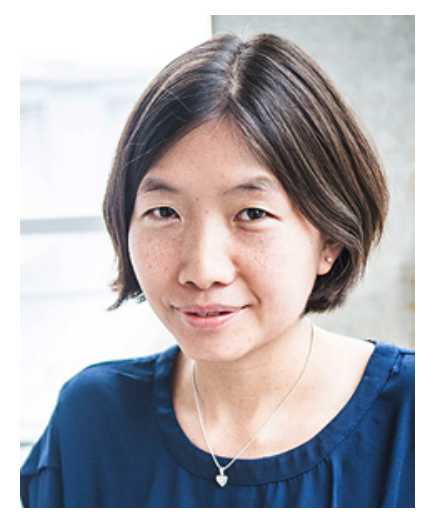

Dr. Natasha Chang

Dr. Natasha Chang is an Assistant Professor in the Department of Biochemistry at McGill University. She obtained her PhD with Gordon Shore at McGill University and performed her postdoctoral training with Michael Rudnicki at the Ottawa Hospital Research Institute. Her research has identified a role for the dystrophin glycoprotein protein complex in regulating muscle stem cell fate, which has led to enhanced understanding of how muscle stem cell functions are impaired in Duchenne muscular dystrophy. She started her lab in August 2019 and her group is focused on determining how muscle stem cells control their fate and how muscle stem cell dysfunction contributes to muscle disease.

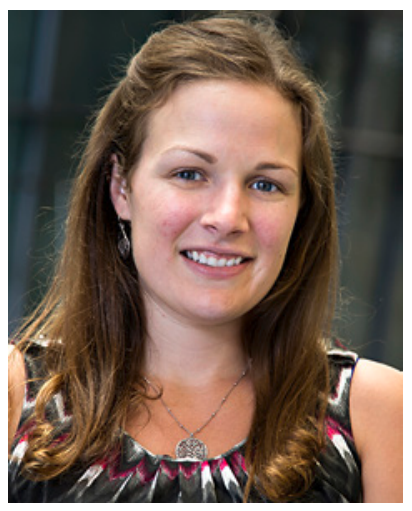

Courtney Dumont

Courtney Dumont joined the department of Biomedical Engineering at the University of Miami in 2018 where her group, Neural Stem and Tissue Engineered Microenvironments (NeuSTEM), works at the interface of neural, vascular, and immune engineering to overcome barriers that arise after injury to the nervous system. She has secondary appointments in the Department of Biochemistry and Molecular Biology and the John T. Macdonald Biomedical and Nanofabrication Institute at the University of Miami, which has allowed the NeuSTEM lab to expand its therapeutic toolkit to include drug delivery platforms from the nano- to micrometer scale. In 2020, Dumont was awarded the inaugural Junior Frost Fellow Award in chemistry for her targeted drug delivery strategies following nerve injury. Her lab continues to develop new biomaterial, stem cell, and drug delivery strategies that address intrinsic and extrinsic barriers to spinal cord repair and works closely with members of the Miami Project to Cure Paralysis to continue to redefine our understanding of the pathophysiology after injury and identify new therapeutic targets. 


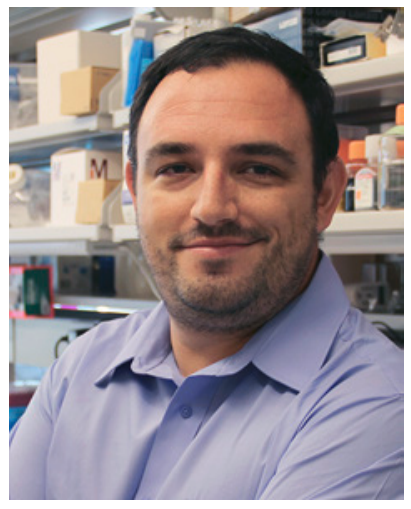

Dr. Riccardo Gottardi

Dr. Riccardo Gottardi is an Assistant Professor of Pediatrics and of Bioengineering at University of Pennsylvania. Dr. Gottardi leads the Bioengineering and Biomaterials Laboratory at the Children's Hospital of Philadelphia, working closely with clinicians to develop engineering solutions for pediatric airway disorders focusing on translational tissue engineering and on novel preventative drug delivery approaches with accelerated pathway to the clinic. His lab investigates the basic cellular, molecular, and microbiological mechanisms as well as the genetic aspects of ear, nose, and throat damage and healing, and combines the development of organ-on-chip and animal models of cartilage, vocal folds, and craniofacial tissues to study growth, disease, and repair. Dr. Gottardi's research interests include tissue engineering of the musculoskeletal system, regenerative rehabilitation for cartilage regeneration, modelling osteoarthritis and osteochondral crosstalk via organ-on-chip culture for the development of new drug, decellularized matrix approaches to tissue repair, airway engineering, and the influence of the microbiome on airway disorders and repair.

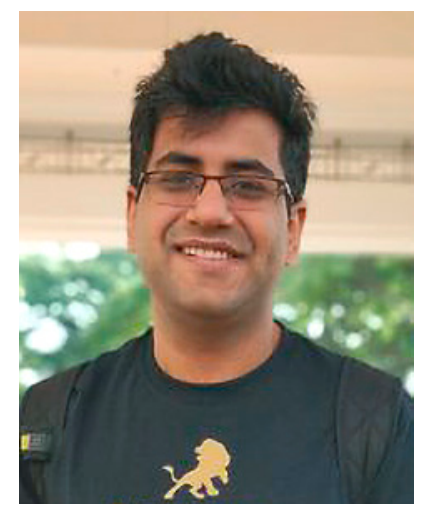

Dr. Mohit Kumar Jolly

Dr. Mohit Kumar Jolly (Current Position: Assistant Professor, Centre for BioSystems Science and Engineering, Indian Institute of Science, Bangalore 560012 India) leads the Cancer Systems Biology group at the Centre for BioSystems Science and Engineering, Indian Institute of Science. He has made seminal contributions to decoding the emergent dynamics of epithelial-mesenchymal plasticity (EMP) in cancer metastasis, through mathematical modeling of regulatory networks implicated in EMP; his work has featured on the cover of Journal of Clinical Medicine, Cancer Research, and Molecular and Cellular Biology, and he won the 2016 iBiology Young Scientist Seminar Series - a coveted award for communicating one's research to a diverse audience. Currently, his lab focuses on decoding mechanisms and implications of non-genetic heterogeneity in cancer metastasis and therapy resistance, with specific focus on mechanism-based and data-based mathematical modeling in close collaboration with experimental cancer biologists and clinicians. $\mathrm{He}$ is an elected fellow of Indian National Young Academy of Sciences (INYAS), and currently serves as the Secretary of The International Epithelial-Mesenchymal Transition Association (TEMTIA).

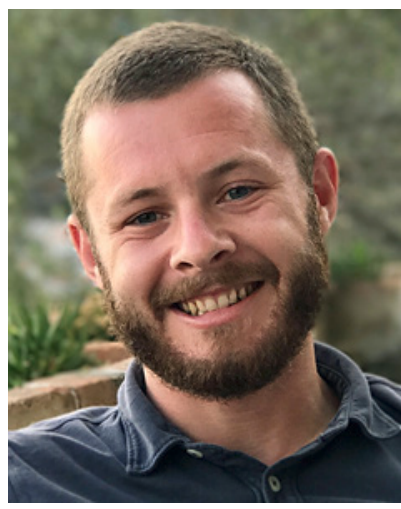

Ryan Stowers

Ryan Stowers is an Assistant Professor at the University of California, Santa Barbara in the Department of Mechanical Engineering and the Center for Bioengineering. Dr. Stowers obtained his Ph.D. from the University of Texas at Austin and then was a National Institutes of Health Ruth L. Kirschstein Postdoctoral Fellow at Stanford University. His laboratory explores how cells interact with and are influenced by their environment by engineering 3D hydrogel cell culture platforms to recapitulate influential features of the cellular microenvironment. 


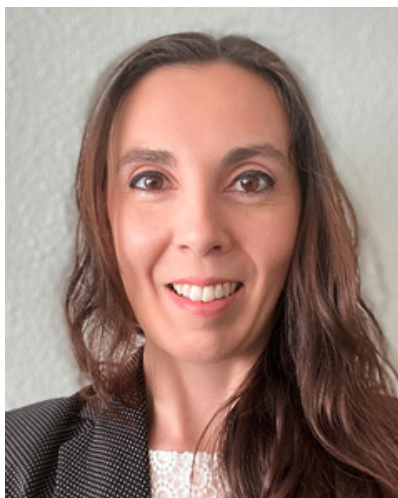

Dr. Liliana Moreira Teixeira

Dr. Liliana Moreira Teixeira is an Assistant Professor with a shared position between the department of Developmental BioEngineering, University of Twente, and the department of Veterinary Sciences / Regenerative Medicine Utrecht, Utrecht University, The Netherlands. Liliana is a biomedical engineer with a strong background in (micro-) tissue engineering, who is interested in developing advanced microfluidic (patho-) physiological in vitro models for drug screening and personalized medicine approaches. Liliana is particularly interested in using organon-a-chip technology to investigate the role of organ communication, mechanobiology and inflammation, in disease processes, such as osteoarthritis, rheumatoid arthritis, and cancer. Dr. Liliana Moreira Teixeira is also the scientific co-director of the Organ-on-Chip Center Twente, a recently established centre of expertise, which aims at bringing together researchers interested in Organ-on-Chip and share their knowledge.

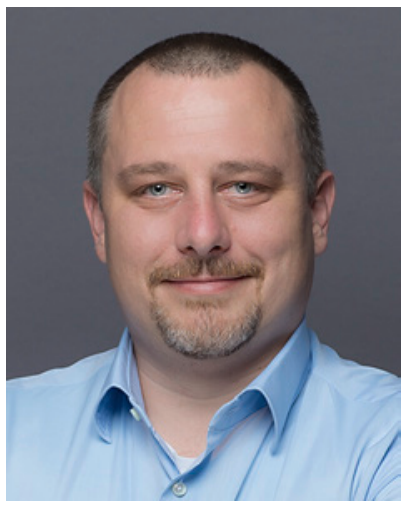

Peter Walentek

Peter Walentek studies tissue formation and remodeling in development and disease using animal models (Xenopus frogs and mice) and stem cell cultures of airway epithelia. He is an Emmy-Noether group leader at the University Freiburg Medical Center and associated PI at the Albert-Ludwigs-University Freiburg excellence cluster Centre for Integrative Biological Signalling Studies (CIBSS). He was a recipient of multiple awards, including a NIH Pathway to Independence award, an UC Berkeley Department of Molecular and Cell Biology outstanding postdoc award, and a $\mathrm{PhD}$ thesis award from the Universitätsbund Hohenheim. His major contributions include the establishment of the Xenopus embryonic epidermis as model for mucociliary disease research, elucidating the roles of Wnt signaling in the regulation of ciliated and stem cells, and work on the post-transcriptional regulation of cilia. 